\title{
Violation and persistence of the K-quantum number in warm rotating nuclei
}

\author{
M. Matsuo ${ }^{1}$, T. Døssing ${ }^{2}$, A. Bracco ${ }^{3}$, G.B. Hagemann ${ }^{2}$, \\ B. Herskind ${ }^{2}$, S. Leoni ${ }^{3}$, E. Vigezzi ${ }^{3}$ \\ ${ }^{1}$ Graduate School of Science and Technology, Niigata University, Niigata 950-2181, Japan \\ ${ }^{2}$ Niels Bohr Institute, University of Copenhagen, DK2100 Copenhagen Ø, Denmark \\ ${ }^{3}$ INFN sez. Milano, and Department of Physics, University of Milano, Milan 20133, Italy
}

\begin{abstract}
The validity of the K-quantum number in rapidly rotating warm nuclei is investigated as a function of thermal excitation energy $U$ and angular momentum $I$, for the rare-earth nucleus ${ }^{163} \mathrm{Er}$. The quantal eigenstates are described with a shell model which combines a cranked Nilsson mean-field and a residual two-body interaction, together with a term which takes into account the angular momentum carried by the K-quantum number in an approximate way. K-mixing is produced by the interplay of the Coriolis interaction and the residual interaction; it is weak in the region of the discrete rotational bands $(U \lesssim 1 \mathrm{MeV})$, but it gradually increases until the limit of complete violation of the K-quantum number is approached around $U \sim 2-2.5 \mathrm{MeV}$. The calculated matrix elements between bands having different K-quantum numbers decrease exponentially as a function of $\Delta K$, in qualitative agreement with recent data.
\end{abstract}

PACS: 21.10.Re, 21.60.-n, 23.20.Lv, 24.60.Lz, 25.70.Gh, 27.70+q

Keywords: K-quantum number, compound states, warm rotating nuclei, band crossings, residual interaction, quasi-continuum gamma spectra.

Corresponding author:

Masayuki Matsuo

Graduate School of Science and Technology, Niigata University

Ikarashi Ninocho, Niigata 950-2181, Japan

e-mail: matsuo@nt.sc.niigata-u.ac.jp

telefax: +81-25-263-3961 


\section{INTRODUCTION}

Recent experimental studies of the $\gamma$-decay following fusion reactions have been able to explore in great detail the $\gamma$-ray spectra of well deformed nuclei. For example, in the case of the axially symmetric nucleus ${ }^{163} \mathrm{Er}$, about 15 rotational bands have been resolved up to rotational frequency around $500 \mathrm{keV}$ [1], and it has been confirmed that the Kquantum number [2] is a basic quantity in classifying the rotational bands. These bands cover a region of rather low energy above the yrast line, and it has been possible to classify interactions at band crossings in relation to the differences between the K-quantum number of the crossing bands [3]. It was found that significant K-selection rules still exist around angular momentum $I \sim 25$, although much weaker than those valid at low $I$ close to the band heads.

With the same experiments, one can also analyze the quasicontinuum spectra of $\gamma$-rays emitted from states of somewhat higher thermal excitation energy [3-6]. In this case, one can probe the feeding into specific configurations by gating on selected low lying transitions. In particular, the study of the covariance of spectra gated by high-K and low-K bands has indicated a certain persistence of K-selection rules [7]. As it has been clearly stated by B. Mottelson [8], the question of K-quantum number violation in thermally excited states is a key issue in the study of the transition between ordered and chaotic many-nucleon motion

caused by the residual interaction and high level density [9-11]. The information gathered by fusion experiments supplements in an important way that available through the study of the $\gamma$-decay from neutron resonances.

Generally, the complex nature of states at neutron resonances may amplify symmetry breaking, parity violation being the most striking example [9]. Theoretical estimates based on the Gaussian Orthogonal Ensemble model [12] and the Coriolis force definitely predict a complete K-violation at the neutron resonance states in the case of neutron resonances $[13,14]$. The model applied in this paper is in line with this prediction, since we find that our results can be reasonably well described by the GOE, for thermal excitation energy $U$ above 
about $2 \mathrm{MeV}$, both with regard to rotational transition matrix elements and level spacings [15-17]. On this basis, it is indeed surprising that an experimental analysis [18] of transitions from neutron resonance states finds significant K-hindrance effects in the $\gamma$-decay following thermal neutron capture. This question of the K-quantum number of neutron resonance states has been discussed from various theoretical viewpoints $[13,19,20]$.

In the present paper, we give a theoretical description of the K-quantum number in rotating nuclei, focusing on the evolution with increasing angular momentum and heat energy, presenting results for the nucleus ${ }^{163} \mathrm{Er}$.

We formulate our description on the basis of the cranked-shell model that has been previously developed to describe the rotating compound states and the associated rotational damping phenomena [21], including a two-body residual interaction. However, we shall introduce a modification to the standard cranking hamiltonian, that allows a realistic description of high-K states. The cranking, or Coriolis, term provides a weak breaking of the K-quantum number. This weak breaking is in turn amplified by the mixing between bands, caused by the residual interaction. It is to be noted that the residual interaction we have adopted would not by itself induce any K-mixing.

\section{CRANKED SHELL MODEL AND HIGH-K STATES}

\section{A. Cranked mean field and residual interaction}

Assuming that an axially deformed nucleus rotates about a principal axis (usually taken as the $x$-axis) with a constant rotational frequency, the standard cranking model [22] can describe the rotational bands associated to a given intrinsic configuration. We start with the cranked Nilsson single-particle Hamiltonian $h_{c r}=h_{N i l s s o n}-\omega_{x} J_{x}$. The rotational frequency $\omega_{x}$ is chosen so that the mean expectation value of the angular momentum $J_{x}$ along the

rotational $x$-axis is equal to the spin $I$. Many-particle many-hole $(n \mathrm{p}-n \mathrm{~h})$ configurations in the cranked Nilsson Hamiltonian are produced with use of the common rotational frequency 
$\omega_{x}(I)$, and adopted as an unperturbed shell model basis for the spin $I$, which we denote by $|\mu(I)\rangle$. The basis states are regarded as unperturbed rotational bands. Since the angular momentum variable in the cranking model is the component $I_{x}$ along the rotational $x$-axis, we can construct the laboratory energy $E_{\mu}(I)=E_{\mu}\left(I_{x}\right)$ of the basis state $\mu$ by identifying the angular momentum along the rotational $x$-axis $I_{x}=\left\langle\mu\left|J_{x}\right| \mu\right\rangle$ with the total angular momentum $I$. The laboratory energy $E_{\mu}(I)$ is a sum of occupied cranked-Nilsson routhian energies, the Strutinsky correction with the rotating liquid drop energy, and the energy correction due the transformation to the laboratory frame. In addition, we introduce a residual interaction among the basis configurations, adopting the surface-delta interaction (SDI) [23]. The shell model Hamiltonian thus constructed at spin $I$ reads

$$
H_{\mu \mu^{\prime}}(I)=E_{\mu}(I) \delta_{\mu \mu^{\prime}}+V_{\mu \mu^{\prime}}(I),
$$

where $V_{\mu \mu^{\prime}}(I)$ is the matrix elements of the SDI. The residual interaction is essential to describe the compound states at finite thermal energy and the rotational damping since both are caused by mixing among the basis configurations [24]. The states near the yrast line, on the other hand, are less influenced by the residual interaction, and they survive as discrete rotational bands. The details of the cranked shell model Hamiltonian (1) are given in Ref. [21].

\section{B. Hamiltonian including the K-quantum number}

In deriving the Hamiltonian (1) we have neglected the K-quantum number by applying the angular momentum relation $I=I_{x}$, which should be modified if the K-quantum number is taken into account.

Let us now discuss how the main effects associated to the K-quantum number can be incorporated. If we consider a rotational band $i$ carrying the K-quantum number $K_{i}$, the angular momentum about the rotational $x$-axis will be given by $I_{x}=\sqrt{I^{2}-K_{i}^{2}}$. Denoting by $E_{i}^{\text {crank }}\left(I_{x}\right)$ the rotational energy acquired by the band cranking about the 
$x$-axis, the rotational energy taking into account the K-quantum number is given by $E_{i, K_{i}}(I)=E_{i}^{\operatorname{crank}}\left(I_{x}=\sqrt{I^{2}-K_{i}^{2}}\right)$. In the case of high spin states, that is $I \gg K_{i}$, or if the dependence on $I_{x}$ is quadratic as expected from a rigid-body rotation, the above equation can be approximated as

$$
E_{i, K_{i}}(I)=E_{i}^{c r a n k}\left(I_{x}=I\right)-K_{i}^{2} / 2 \mathcal{J}_{i}
$$

where $\mathcal{J}_{i}=I_{x}\left(\partial E_{i}^{c r a n k} / \partial I_{x}\right)^{-1}=I_{x} / \omega_{x}$ is the kinematic moment of inertia of the band. Note that the energy correction $-K_{i}^{2} / 2 \mathcal{J}_{i}$ for small angular momenta converges toward the corresponding term in Bohr's collective rotational energy $E_{i, K_{i}}(I)=\left\{I(I+1)-K_{i}^{2}\right\} / 2 \mathcal{J}_{i}+E_{i}^{\text {intr }}$ ( $E_{i}^{\text {intr }}$ being the intrinsic excitation energy) [2]. The first term $E_{i}^{c r a n k}\left(I_{x}=I\right)$ corresponds to our previous cranked shell model Hamiltonian, Eq.(1). The second term $-K_{i}^{2} / 2 \mathcal{J}_{i}$ represents the effect of the K-quantum number on the rotational energy, which we want to include in the present calculations.

In accordance with these considerations, we modify the shell model Hamiltonian as

$$
H_{\mu \mu^{\prime}}(I)=E_{\mu}(I) \delta_{\mu \mu^{\prime}}+V_{\mu \mu^{\prime}}(I)-\left(J_{z}^{2}\right)_{\mu \mu^{\prime}} / 2 \mathcal{J}_{\mu \mu^{\prime}}
$$

The last term is an operator form of the energy correction $-K_{i}^{2} / 2 \mathcal{J}_{i}$, where $J_{z}$ is the angular momentum operator of the constituent nucleons along the symmetry axis $z$, and $\mathcal{J}_{\mu \mu^{\prime}}=$ $\left(\mathcal{J}_{\mu}+\mathcal{J}_{\mu^{\prime}}\right) / 2$ is the kinematic moment of inertia of the np-nh basis configurations. Note that $J_{z}^{2}$ has off-diagonal matrix elements $\mu \neq \mu^{\prime}$ since the unperturbed states $|\mu(I)\rangle$ do not necessarily have a good K-quantum number. The new term in the Hamiltonian favors the states with high values of the K-quantum number, as will be illustrated below. Appendix A describes how high-K (or rather high- $\mathrm{K}^{2}$ ) states are generated from the signature basis by the Hamiltonian (3).

It is possible to relate our cranked shell model to the tilted-axis cranked mean-field (TAC) model [25], which has been used to describe the high-K rotational bands near the yrast line. If one makes a mean-field approximation to the $J_{z}^{2}$-term, it reads $J_{z}^{2} / 2 \mathcal{J} \sim\left(\left\langle J_{z}\right\rangle / \mathcal{J}\right) J_{z}=\omega_{z} J_{z}$, which corresponds to the second part of the generalized cranking term $\omega_{x} J_{x}+\omega_{z} J_{z}$ in the 
TAC model. Note however that the self-consistency between the directions of the angular momentum vector and the rotational frequency vector, required by the tilted rotation, is achieved approximately but not exactly in the present model since a common cranking rotational frequency $\omega_{x}$ is ascribed in our case for all the states at a given spin. This approximation affects also the energy eigenstates obtained by diagonalizing the Hamiltonian (3). The TAC mode is more consistent in this respect since the self-consistency is achieved for each rotational band. Our treatment enables, on the other hand, a description of configuration mixing in compound states caused by the residual two-body interaction.

\section{MIXING OF K-QUANTUM NUMBER}

\section{A. Effects on low-lying bands}

Numerical calculations have been performed for ${ }^{163}$ Er. In the calculations, we employ $4000 \mathrm{np}$-nh basis states with the lowest excitation energies to diagonalize the Hamiltonian (1) for each $I^{\pi}$. The truncation corresponds to a cut-off in excitation energy of approximately $4 \mathrm{MeV}$. This larger basis (compared to Ref. [21]) is necessary to produce the high-K states whose energy is lowered by the $J_{z}^{2}$-term after the basis selection has been made. The deformation parameters $\epsilon_{2}=0.252$, and $\epsilon_{4}=-0.004$ are taken from the tilted axis cranking analysis of the same nucleus [26], while the other model parameters are the same as those of Ref. [21].

The energy levels calculated in the spin range $I=20-60$ are shown in Fig.1, where the strong stretched E2 transitions forming rotational band structures are evidenced. Many of the states whose excitation energy $U$ relative to yrast is smaller than about $1 \mathrm{MeV}$ form rotational bands whereas the rotational band structure gradually disappears as $U$ becomes larger than $\sim 1 \mathrm{MeV}$, indicating that the rotational damping sets in around this excitation

energy. We can define the average value of $K$ for each energy level $i$ by $K_{i}=\sqrt{\left\langle i\left|J_{z}^{2}\right| i\right\rangle}$ in terms of the expectation value of $J_{z}^{2}$. It is found that many high-K states with a large 
value of $K_{i}$, e.g., $K_{i}>8$, are produced. Examples for the positive parity states are shown in Fig.2(a). The lowest energy positive parity high-K states at excitation energy $U \approx$ 0.4MeV form a rotational band extending up to $I \sim 89 / 2$ with essentially zero signature splitting. It has a large value of $K_{i}, K_{i} \approx 10$, and its eigenfunction is predominantly $(n[523] 5 / 2)(p[404] 7 / 2)(p[523] 7 / 2)^{-1}$ with the combination of $K=5 / 2+7 / 2+7 / 2=19 / 2$. The role of the new $J_{z}^{2}$-term in the Hamiltonian (3) can be appreciated comparing Fig.2(a) to 2(b), where the $J_{z}^{2}$-term is neglected, disregarding the contribution of the K-quantum number to the angular momentum. As a consequence, in Fig. 2(b) there are essentially no low-lying high-K states. Fig.2(c) shows the np-nh mean-field basis bands, where both the residual interaction and the $J_{z}^{2}$-term are neglected. Eight np-nh basis bands located at $E-I(I+$ 1) $/ 148 \approx 0.8 \mathrm{MeV}$ at $\operatorname{spin} I \sim 20-25$ exhibit an approximate octet degeneracy, arising from the $(n[523] 5 / 2)(p[404] 7 / 2)(p[523] 7 / 2)^{-1}$ configurations where each cranked Nilsson orbit has approximate two-fold degeneracy with different signatures. The $J_{z}^{2}$-term resolves the octet degeneracy and produces the lowest high-K state $K_{i} \approx 19 / 2$ in Fig.2(a). The energy shift due to the $J_{z}^{2}$-term amounts to several hundreds of keV.

The lowest high-K band in Fig.2(a) corresponds to the positive parity high-K rotational band (faE/eaE) observed in the experiments (Fig.2(d)), for which a similar quasiparticle configuration and $K=19 / 2$ have been assigned [1]. The calculated excitation energy of this band relative to yrast is about $350-500 \mathrm{keV}$, which is in fair agreement with the experiment concerning the highest spin region $I \approx 30-36$. The second positive parity high-K band (YAG/XAG) observed in the experiment has an excitation energy $U=1.1-1.3 \mathrm{MeV}$. In the calculation, a second excited high-K bands with positive parity lies around $U=$ $0.8-0.9 \mathrm{MeV}$, showing that we here have an overall description of the high-K bands near yrast. However, specific properties of individual rotational bands, including both high-K and low-K bands, are not well reproduced. The calculated second high-K band has slightly different configurations from that of the observed one while both involve the deformationaligned neutron orbit $(n[505] 11 / 2)$. Also, alignments of $\mathrm{i}_{13 / 2}$ quasi-neutrons and $\mathrm{h}_{11 / 2}$ quasiprotons observed in the experiments are missing. These disagreements may be traced back 
mostly to the insufficient treatment of pairing correlations, which are only partly taken into account through the shell model diagonalization in the present model. A pairing gap of $0.8-0.9 \mathrm{MeV}$ is adopted both for neutrons and protons in the tilted axis cranking (TAC) calculation [26], which provides a better description of near-yrast rotational bands. Note also that the TAC treats the self-consistency in the rotational angular momentum vector more precisely.

It is noted that deformation aligned single-particle orbits having sizable value of $\Omega_{i}$ (the approximate cranked Nilsson quantum number of $J_{z}$ ) lying near the Fermi surface play important roles to produce high-K states. Their contribution depends on the neutron and proton numbers, and deformations. Concerning the neutrons, the orbits $n[523] 5 / 2$ and $n[505] 11 / 2$ are relevant. If we consider slightly heavier nuclei around ${ }^{168} \mathrm{Yb}$, contributions from these orbits are less effective, and the same cranked shell model produces a smaller number of high-K states.

\section{B. K-mixing as a function of thermal excitation energy and spin}

Fig.3(a) plots the value of $K_{i}$ for the energy levels up to $U=2 \mathrm{MeV}$ at $I^{\pi}=61 / 2^{+}$. The energy levels are marked with different symbols depending on whether they are connected with strong E2 transitions to spin $I-2$ or $I+2$ (cf. Fig.1). There exist several high-K rotational bands with $K \approx 10-15$ below $U \sim 1.5 \mathrm{MeV}$ (See also Fig.2(a)). If the $J_{z}^{2}$-term is neglected (Fig.3(b)), high-K states with $K>10$ are not produced.

Fig.3(c) displays the results obtained without the residual two-body interaction (i.e. only the unperturbed cranked-Nilsson energy and the $J_{z}^{2}$-term are considered). Essentially, each solution obtained without the residual interaction forms a rotational band, and each band has a value of $K_{i}$ which changes only little as the spin changes. We call these solutions the $K$-basis bands (and denote by the letter $|\kappa\rangle$ ) since they represent unperturbed rotational bands where the K-quantum number is taken into account but the residual interaction is neglected. Over the whole energy region covered in Fig.3(c), the value of $K_{i}$ for the K-basis 
bands fluctuates from state to state within a wide range, reaching values up to $K \sim 15$ (note that $K_{i}$ is positive by definition).

With the residual interaction included (Fig.3(a) vs. Fig.3(c)), the number of high-K states with $K>10$ decreases in the high excitation region $U>1.5 \mathrm{MeV}$. In fact, when the mixing becomes strong, the basis bands are mixed more and more democratically and the values of $K_{i}$ of individual eigenstates tend to converge around an average value $K \sim 7$, with increasing $U$ as is seen in Fig.3(a).

It is possible to describe the K-mixing more quantitatively. For this purpose we first consider a quantity which measures the degree of K-mixing within the individual energy levels. Such a quantity may be defined in terms of the variance of the operator $J_{z}^{2}$ for a given energy eigenstate $|i\rangle$, which is equal to $\left\langle i\left|J_{z}^{4}\right| i\right\rangle-\left\langle i\left|J_{z}^{2}\right| i\right\rangle^{2}$. We denote the average value of this quantity by $\sigma^{2}\left(K^{2}\right)_{\text {mix }}=\overline{\left\langle i\left|J_{z}^{4}\right| i\right\rangle-\left\langle i\left|J_{z}^{2}\right| i\right\rangle^{2}}$, where the bar implies averaging over $i^{\prime}$ s in an energy bin.

On the other hand, each energy level has a different value of $K_{i}$ as can be seen from the distribution of $K_{i}$ shown in Fig.3(a). We then consider the variance of this statistical fluctuation of $K_{i}$ among the different states $|i\rangle, \sigma^{2}\left(K^{2}\right)_{\text {stat }}=\overline{\left\langle i\left|J_{z}^{2}\right| i\right\rangle^{2}-\overline{\left\langle i\left|J_{z}^{2}\right| i\right\rangle}}$.

Using these variances, a quantitative measure of the degree of K-mixing can be given by the ratio

$$
r_{K m i x}=\frac{\sigma^{2}\left(K^{2}\right)_{\text {mix }}}{\sigma^{2}\left(K^{2}\right)_{\text {mix }}+\sigma^{2}\left(K^{2}\right)_{\text {stat }}}
$$

between the variances of the intrinsic K-mixing and total K-quantum number fluctuation. If the K-mixing is weak, the ratio $r_{K m i x}$ will attain a value close to zero. If many different $\mathrm{K}$ quantum numbers are mixed strongly into individual levels in a random manner, $\sigma^{2}\left(K^{2}\right)_{m i x}$ dominates and the ratio will take a value close to unity. As a guideline, one may define regions of weak K-mixing by $r_{K \text { mix }}<0.5$, and strong K-mixing by $r_{K m i x}>0.8$. In accordance with the discussion of rotational damping in terms of the average branching number, with the value $n_{\text {branch }} \approx 2$ defining the energy for onset of damping, an equivalent condition for the onset of K-mixing should be $r_{K m i x} \approx 0.5$ (see Appendix B). 
Fig.4(a) shows the two variances $\sigma^{2}\left(K^{2}\right)_{\text {mix }}$ and $\sigma^{2}\left(K^{2}\right)_{\text {stat }}$, and the total variance of the K-quantum number as functions of $U$ for the states at spins $I=\frac{59}{2}, \frac{61}{2}$. The K-mixing ratio calculated from these variances is plotted in Fig.4(b). The figure displays an onset of the K-mixing (as defined by $r_{K m i x} \approx 0.5$ ) around $U \approx 1.5 \mathrm{MeV}$, followed by a monotonic approach towards complete K-mixing with increasing $U$. It was noted above that the onset of rotational damping takes place around $U \approx 1.0 \mathrm{MeV}[4,5]$. This onset energy is predicted by calculations $[24,21]$ and observed in experiments in the rare earth nuclei around ${ }^{168} \mathrm{Yb}$. For $I=\frac{59}{2}, \frac{61}{2}, \mathrm{~K}$-mixing sets in at higher energies than rotational damping. Also, one can observe in Fig.3(a) that several high-K rotational bands remain discrete in the energy region $U=1.0-1.5 \mathrm{MeV}$, where most low-K states do not form rotational bands due to the rotational damping. Thus, it appears that the delayed onset of K-mixing, relative to the onset of damping, is also accompanied by a delayed onset of damping of high-K bands.

To obtain an overall view, the K-mixing ratio $r_{K m i x}$ is shown in Fig.5(a) as a function of spin and thermal excitation energy $U$. It is seen that the K-mixing ratio increases from $r_{\text {Kmix }} \approx 0.2$ to $\approx 0.9$ as $U$ increases from zero to $U \approx 2 \mathrm{MeV}$, and that the amount of K-mixing increases with increasing angular momentum. The statistical limit $\left(r_{K m i x} \rightarrow 1\right)$, is gradually approached for higher thermal energies, $U>2 \mathrm{MeV}$.

The delayed onset of K-mixing relative to damping displayed by figures 3(a) and 4(a) is seen only at low angular momenta $I \leq 35 \hbar$. The fact that K-mixing is rather weak even for rather high energy $U<1.5-1.8 \mathrm{MeV}$ at the lower angular momenta may provide a first explanation of the experimental observation [7] of the persistence of the K-quantum number in the quasi-continuum spectrum observed after a fusion reaction.

The role of the residual interaction in the K-mixing should be stressed. In our calculations we have adopted the so-called surface delta interaction (SDI), which respects the K-quantum number. Thus, the Coriolis term is essential in order to induce K-mixing. On the other hand, without the residual interaction, the K-mixing is very weak over the whole region considered, as can be seen in Fig.5(b), which corresponds to the K-basis bands of Fig.3(c). The Coriolis term alone only produces a weak K-mixing, gradually increasing towards the very high spins 
$I=40-60$. In other words, the strong K-mixing $(U>2 \mathrm{MeV})$ in Fig.5(a) is a consequence of the interplay between the two-body residual interaction, which is responsible for the orderto-chaos transition, and the Coriolis interaction which by itself would induce only a modest violation of the axial symmetry.

To further investigate the role of the two-body interaction, we show in Fig.5(c) the result of a calculation where the SDI is replaced by a random two-body interaction. In this case, the two-body matrix elements are given by Gaussian random numbers with the r.m.s. value of $12 \mathrm{keV}$. This r.m.s. value is chosen to reproduce the same onset energy of rotational damping as the SDI, whose matrix elements have the r.m.s. value of $19 \mathrm{keV}$ [21]. The Kmixing and its energy dependence are much stronger than for the SDI interaction; K-mixing sets in already at $U \sim 0.5 \mathrm{MeV}$, that is below the onset of rotational damping, and strong $\mathrm{K}$-mixing is already achieved at a much lower energy $U \sim 1.2 \mathrm{MeV}$. The delayed onset of K-mixing seen at the low spins disappears with the random interaction. These differences between the SDI and the random interaction can be related to the fact that the SDI in itself respects the K-quantum number, so the Coriolis interaction is needed to induce K-mixing. Conversely, the K-quantum number is completely ignored by the random interaction, and

in this case, the degree of $\mathrm{K}$-mixing is approximately given by the average number $N_{\text {mix }}$ of components of the wave function, as $r_{K m i x} \approx 1-\frac{1}{N_{m i x}}$ (cf. Appendix B).

\section{K-SELECTION IN INTERACTION MATRIX ELEMENTS}

For resolved bands of low thermal excitation energy, some interaction matrix elements may be determined at band crossings of pairs of bands having different rotational frequency. Recently, such experimental interaction matrix elements were extracted from an analysis of band crossings in ${ }^{163} \mathrm{Er}$ [1] and its neighbors. In order to investigate K-selection rules associated to these matrix elements, one may assign the K-value from the band head spin, and plot the extracted interaction strength versus the difference $\Delta K$ in $\mathrm{K}$-quantum numbers for the crossing bands. (One can justify this assignment by relating to our cranked shell 
model, where we find that the K-values calculated in the cranked shell model are not identical to the band head K-values, but they are on the other hand not very different for the unmixed rotational bands at the lowest heat energies.)

The result obtained from the 32 band crossings in ${ }^{163} \operatorname{Er}[1]$ and neighboring nuclei ${ }^{162} \mathrm{Tm}$ [27], ${ }^{163} \mathrm{Tm}[28]$, and ${ }^{164} \mathrm{Lu}[29]$ is shown in Fig.6(a). Nineteen crossings can be related to a two-body residual interaction, and a previous analysis limited to them [3] already found that they correspond to an average interaction strength of $14 \mathrm{keV}$, and that they are correlated with $\Delta K$. The other crossings include those caused by the changes in the pair-field with increasing rotational frequency, which are not well described in the present model. The distribution of the the experimental matrix elements suggests an exponential scaling

$$
V_{K K^{\prime}} \propto \exp \left(-\left|K-K^{\prime}\right| / \delta K_{0}\right)
$$

with respect to the difference in $\mathrm{K}$ between the crossing bands, and from figure 6(a) one may roughly read off an effective K-correlation interval $\delta K_{0} \approx 1.6$.

We compare the experimental matrix elements to matrix elements of the SDI among the energy eigenstates in the region of resolved bands. Fig.6(b) plots the matrix elements $V_{i j}$ calculated for all pairs of the lowest 10 energy levels for each $I^{\pi}$, approximately corresponding to an interval in thermal energy $U=0-1 \mathrm{MeV}$. Comparing panels (a) and (b) of the figure, one sees that the calculations are in accordance with the main features of the experiment: (i) : a median around $\sim 20 \mathrm{keV}$ at $\Delta K=0,($ ii $)$ a considerable fluctuation among matrix elements at the same $\Delta K$, and $(i i i)$ an overall exponential decrease with increasing $\Delta K$. For the calculated low lying energy eigenstates, we find a somewhat weaker K-selection rule than that displayed by the experimental band crossings, corresponding to a wider K-correlation interval $\delta K_{0} \approx 2.1$.

The experimental matrix elements are extracted from energy level repulsion and E2 branching ratio between two crossing bands while the theoretical values plotted in Fig.6(b) are the matrix elements calculated directly from the energy eigenstates, which sometimes become a mixture of rotational bands. The small difference in the K-correlation extracted 
from Figs.6(a) and (b) could in part originate from the different methods adopted to extract the matrix elements.

As another possible evaluation of matrix elements, one can also consider the matrix elements of the SDI residual interaction among the lowest energy K-basis states. This is done in fig.6(c). The calculated matrix elements with the K-basis display the same dispersion for fixed $\Delta K$, and a more steep slope with $\Delta K$, corresponding to a correlation interval $\delta K_{0} \approx 1.5$. Nevertheless, both the K-correlation intervals calculated by the two different definitions agrees fairly well with the experimental value.

Since it is the Coriolis force that eventually breaks the K-quantum number, the above K-selectivity of the matrix elements becomes weaker as the spin increases. For very high spins $I=50-58$, the K-correlation interval is found to be $\delta K_{0} \sim 3-4$. Altogether, one expects a linear correspondence between $\delta K_{0}$ and the angular momentum for the low-lying bands. Going to the opposite extreme, the low angular momenta close to the band heads, the typical K-forbiddeness factor [2] of $10^{-2}$ per unit change of $K$ of transition strengths translates into a K-forbiddeness factor of $10^{-1}$ for matrix elements, and thereby $\delta K_{0} \sim 0.4$.

\section{EFFECTIVE NUMBER OF BANDS}

The present model calculations should also address the results found in quasicontinuum spectra gated on specific low-lying bands having a well-defined K-quantum number [7]. However, a quantitative comparison between theory and experiments requires a detailed description of the $\gamma$-cascades. This will be attempted in a future publication, in which a comparison can be made to new quasicontinuum data. The mixing coefficient $r_{K m i x}$ will be an important ingredient also for understanding the K-selection rules displayed by the cascades.

Here, we restrict ourselves to the number of paths $N_{\text {path }}$ associated to low-K or high$\mathrm{K}$ bands. These quantities can be extracted by a fluctuation analysis from the ridge part

of the two-dimensional $E_{\gamma 1} \times E_{\gamma 2}$ spectra. $N_{\text {path }}$ represents an effective number of regular 
rotational bands which are populated by the gamma-cascades.

As indicated in Fig.1, the number of discrete regular rotational bands present in the nucleus will be rather small because of rotational damping. A regular rotational band which gives rise to counts on the ridge in two-dimensional spectra should contain two strong consecutive transitions, obeying rotational energy correlations, that is they should be separated by about $4 / \mathcal{I}, \mathcal{I}$ being a typical band moment of inertia. In our calculations, the number of bands $N_{\text {band }}$ at a given spin is defined as the total number of levels for which $n_{\text {branch }}<2$ [21]. Here, $n_{\text {branch }}=\left(\sum_{j} s_{i j}^{2}\right)^{-1}$ is an effective number of E2 decay-branches where $s_{i j}$ is denotes the normalized E2 transition strength for going from the level $i$ at spin $I$ to the level $j$ at spin $I-2$.

The calculated values of $N_{\text {band }}$ summing over four configurations of parity and signature are shown in Fig.7. In order to have some insight in the role played by the K-quantum number, we also separately evaluate $N_{\text {band }}$ associated with high-K and low-K states, which are classified with the values of $K_{i}=\sqrt{\left\langle i\left|J_{z}^{2}\right| i\right\rangle}$. The total $N_{\text {band }}$ is around $\approx 30-40$ except for $I<30$. This is consistent with the experimental value of $N_{\text {path }} \approx 40$ [7] obtained for ungated spectra.

Rare-earth nuclei previously analysed with respect to ridge fluctuations, ${ }^{168} \mathrm{Yb},{ }^{163} \mathrm{Tm}$, and ${ }^{164} \mathrm{Yb}$, were found to possess around $\sim 25$ regular rotational bands [4,5]. It appears that the surplus of bands in ${ }^{163} \mathrm{Er}$ are actually high-K bands, which do not exist in the other nuclei. An experimental value of $N_{\text {path }} \approx 15$ is obtained for the spectrum gated on a high-K band with $K=19 / 2[7]$. Fig.7 shows that the number of low-K bands $(K<8)$ is around 25, and the number of high-K bands $(K>8)$ is around 15. Here the value $K=8$ distinguishing between low- $\mathrm{K}$ and high- $\mathrm{K}$ bands is somewhat arbitrarily taken as the upper limit of $\mathrm{K}$ for the bands calculated without the $J_{z}^{2}$-term, cfr. fig 3(b). Fig.7 also shows the number of bands calculated without the $J_{z}^{2}$-term. It is seen that high-K rotational bands generated by the $J_{z}^{2}$-term increase the total number of bands by about $\sim 10$. 


\section{CONCLUSIONS}

We have discussed the onset of K-mixing in rapidly rotating warm nuclei by means of microscopic cranked shell model calculation performed for the nucleus ${ }^{163} \mathrm{Er}$.

Although the K-mixing due to the Coriolis force alone is generally weak, it is enhanced by the configuration mixing produced by the residual interaction among the cranked meanfield np-nh bands, becoming more pronounced as the level density increases. A quantitative measure of K-mixing can be given by the K-mixing ratio introduced above. For angular momenta up to about $35 \hbar$, the onset of K-mixing takes place around a heat energy of $U \approx 1.5-1.8 \mathrm{MeV}$, while the statistical limit of strong K-mixing is approached only above $U>2 \mathrm{MeV}$. This dependence of K-mixing on excitation energy is related to the K-selectivity of the matrix elements of the two-body residual interaction we have adopted, which seems to be consistent with the matrix elements experimentally observed at the band crossing interactions. The onset of K-mixing is retarded compared to the onset of damping which, for the same surface-delta interaction, occurs around $U \approx 1 \mathrm{MeV}$. At higher angular momenta $I \geq 35 \hbar, \mathrm{K}$-mixing and damping set in around the same heat energy of $U \approx 1 \mathrm{MeV}$. In the case of a purely random interaction, the K-mixing is much stronger, and essentially complete already at $U \approx 1 \mathrm{MeV}$.

Theoretical results from the same type of calculations may in the future be used for an

analysis of the gamma-ray cascades, thereby addressing the persistence of the K-quantum number, found in the experimental analysis of quasi-continuum spectra following fusion reactions $[7]$.

\section{APPENDIX A. HIGH-K STATES IN THE SIGNATURE BASIS}

Our shell model basis $|\mu(I)\rangle$ consists of the single-particle orbits obtained diagonalizing the cranked single-particle Hamiltonian $h_{c r}=h_{N i l s s o n}-\omega_{x} J_{x}$. The Hamiltonian is symmetric with respect to a rotation of angle $\pi$ about the $x$-axis, so that the single-particle orbits have 
the signature as a good quantum number. The shell model basis $|\mu(I)\rangle$ also keeps the signature, but on the other hand, does not have a good K-quantum number, especially for many-particle many-hole configurations. Diagonalization of the $J_{z}^{2}$-term added to $H$ in the cranked shell model Hamiltonian, Eq.(3), is crucial to produce the high-K states.

To illustrate, let us consider a subspace of one-particle one-hole configurations made of two deformation-aligned orbits, which have single-particle K-quantum numbers $\Omega_{p}>0$ and $\Omega_{h}>0$. To each orbit are associated two almost degenerate states $\phi_{i \pm}=\frac{1}{\sqrt{2}}\left(\phi_{i, K=\Omega_{i}} \pm \phi_{i, K=-\Omega_{i}}\right)$ with both signatures. (Here $\phi_{i, K=\Omega_{i}}$ and $\phi_{i, K=-\Omega_{i}}$ carry positive and negative value for the K-quantum number operator $\left.J_{z}.\right)$ Correspondingly, the $1 \mathrm{p} 1 \mathrm{~h}$ configuration has four degenerate cranked shell-model basis states $\phi_{p+} \phi_{h+}^{-1}, \phi_{p-} \phi_{h-}^{-1}=\frac{1}{2}\left(\phi_{p, K=\Omega_{p}} \phi_{h, K=\Omega_{h}}^{-1}+\phi_{p, K=-\Omega_{p}} \phi_{h, K=-\Omega_{h}}\right) \pm$ $\frac{1}{2}\left(\phi_{p, K=\Omega_{p}} \phi_{h, K=-\Omega_{h}}^{-1}+\phi_{p, K=-\Omega_{p}} \phi_{h, K=\Omega_{h}}^{-1}\right) \quad$ and $\quad \phi_{p+} \phi_{h-}^{-1}, \phi_{p-} \phi_{h+}^{-1}=$ $\frac{1}{2}\left(\phi_{p, K=\Omega_{p}} \phi_{h, K=\Omega_{h}}^{-1}-\phi_{p, K=-\Omega_{p}} \phi_{h, K=-\Omega_{h}}^{-1}\right) \pm \frac{1}{2}\left(\phi_{p, K=\Omega_{p}} \phi_{h, K=-\Omega_{h}}^{-1}-\phi_{p, K=-\Omega_{p}} \phi_{h, K=\Omega_{h}}^{-1}\right)$. Note that none of these $1 \mathrm{p} 1 \mathrm{~h}$ basis states has pure $K=\Omega_{p}+\Omega_{h}$ or $K=\left|\Omega_{p}-\Omega_{h}\right|$. Once the $J_{z}^{2}$-term is diagonalized in the subspace, these states are re-coupled to produce eigenstates of $J_{z}^{2}$. These eigenstates receive energy contributions $-J_{z}^{2} / 2 \mathcal{J} \sim-\left(\Omega_{p} \pm \Omega_{h}\right)^{2} / 2 \mathcal{J}$, yielding two energetically favored high-K states $\frac{1}{\sqrt{2}}\left(\phi_{p, K=\Omega_{p}} \phi_{h, K=-\Omega_{h}}^{-1} \pm \phi_{p, K=-\Omega_{p}} \phi_{h, K=\Omega_{h}}^{-1}\right)$ with $K=\Omega_{p}+\Omega_{h}$ and different signature, and two unfavored low-K states $\frac{1}{\sqrt{2}}\left(\phi_{p, K=\Omega_{p}} \phi_{h, K=\Omega_{h}}^{-1} \pm\right.$ $\left.\phi_{p, K=-\Omega_{p}} \phi_{h, K=-\Omega_{h}}^{-1}\right)$ with $K=\left|\Omega_{p}-\Omega_{h}\right|$. The same mechanism applies to many-particle and many-hole configurations, for which the eigenstate with the highest $\mathrm{K}$ configuration $K=\Omega_{p 1}+\Omega_{p 2}+\cdots+\Omega_{h 1}+\Omega_{h 2}+\cdots$ is produced as the most energetically favored state. We have found that most high-K states obtained in the numerical calculation have a similar structure, although the K-quantum number is only approximately conserved due to the Coriolis effect (i.e. to the cranking term). 


\section{APPENDIX B. RANDOM K-MIXING}

We consider here the K-mixing ratio $r_{K m i x}$ in the statistical limit, for which the expansion coefficients of wave functions on K-basis states will be statistically independent. An energy eigenstate is expanded on the K-basis

$$
|i\rangle=\sum_{n} a_{n}\left|\kappa_{n}\right\rangle
$$

with amplitudes $a_{n}$ and the corresponding probabilities $P_{n}=\left|a_{n}\right|^{2} . J_{z}^{2}$ is diagonal in that basis:

$$
\left\langle i\left|J_{z}^{2}\right| i\right\rangle=\sum_{n}\left|a_{n}\right|^{2} K_{n}^{2}=\sum_{n} P_{n} K_{n}^{2}, \quad\left\langle i\left|J_{z}^{4}\right| i\right\rangle=\sum_{n} P_{n} K_{n}^{4}
$$

Averaging over the mixed band states, one obtains

$$
\overline{\left\langle i\left|J_{z}^{4}\right| i\right\rangle}=\overline{\sum_{n} P_{n} K_{n}^{4}}=\overline{K_{b}^{4}}
$$

where the last term denotes averaging over the basis bands. Further, one obtains:

$$
\overline{\left\langle i\left|J_{z}^{2}\right| i\right\rangle^{2}}=\overline{\left(\sum_{n} P_{n} K_{n}^{2}\right)^{2}}=\overline{\sum_{n} P_{n}^{2} K_{n}^{4}+\sum_{n \neq n^{\prime}} P_{n} P_{n}^{\prime} K_{n}^{2} K_{n}^{\prime 2}}=\overline{\sum_{n} P_{n}^{2} K_{n}^{4}}+\left(\overline{\sum_{n} P_{n} K_{n}^{2}}\right)^{2}-\sum_{n}\left(\overline{P_{n} K_{n}^{2}}\right)^{2} .
$$

Here, the statistical independence between the different components of basis bands has been applied.

Defining the average mixing number into the states

$$
\frac{1}{N_{m i x}} \equiv \overline{\sum_{n} P_{n}^{2}}
$$

one obtains the variance of $J_{z}^{2}$ in mixed states:

$$
\sigma^{2}\left(K^{2}\right)_{m i x}=\overline{\left\langle i\left|J_{z}^{4}\right| i\right\rangle}-\overline{\left\langle i\left|J_{z}^{2}\right| i\right\rangle^{2}}=\left(1-\frac{1}{N_{\text {mix }}}\right)\left[\overline{K_{b}^{4}}-{\overline{K_{b}^{2}}}^{2}\right]
$$

The variance of statistical fluctuation of $K^{2}=\left\langle i\left|J_{z}^{2}\right| i\right\rangle$ is obtained by inserting the same results:

$$
\sigma^{2}\left(K^{2}\right)_{\text {stat }}=\overline{\left\langle i\left|J_{z}^{2}\right| i\right\rangle^{2}}-{\overline{\left\langle i\left|J_{z}^{2}\right| i\right\rangle}}^{2}=\frac{1}{N_{\text {mix }}}\left[{\overline{K_{b}^{4}}}^{-{\overline{K_{b}^{2}}}^{2}}\right]
$$


Thus, one finds that the K-mixing ratio, Eq.(4), is given by

$$
r_{K m i x}=1-\frac{1}{N_{m i x}}
$$

In fact, the Coriolis interaction obeys strong selection rules on the matrix elements $\Delta K=$ \pm 1 , and with a residual interaction, which obeys the K-quantum number, one will not expect these statistical considerations to be valid. Rather, they are relevant for a random residual interaction, and there one sees that mixing even a small number of states causes strong K-mixing. Already a two-state mixing $N_{m i x}=2$ corresponds to $r_{K \operatorname{mix}}=0.5$ and $N_{\text {mix }}=5$ is enough to generate strong $\mathrm{K}$-mixing, $r_{K \operatorname{mix}}=0.8$. 


\section{REFERENCES}

[1] G.B. Hagemann, H. Ryde, P. Bosetti, A. Brockstedt, H. Carlsson, L.P. Ekström, A. Nordlund, R.A. Bark, B. Herskind, S. Leoni, A. Bracco, F. Camera, S. Frattini, M. Mattiuzzi, B. Million, C. Rossi-Alvarez, G. de Angelis, D. Bazzacco, S. Lurnardi, M. de Poli, Nucl. Phys. A618 (1997) 199.

[2] A. Bohr, B.R. Mottelson, Nuclear Structure. vol. II (Benjamin, 1975).

[3] T. Døssing, B. Herskind, G.B. Hagemann, A. Harsmann, S. Törmanen, S. Ödegård, M. Matsuo, E. Vigezzi, R.A. Broglia, Nucl. Phys. A649 (1999) 370c.

[4] B. Herskind, A. Bracco, R.A. Broglia, T. Døssing, A. Ikeda, S. Leoni, J. Lisle, M. Matsuo, E. Vigezzi, Phys. Rev. Lett. 68 (1992) 3008.

[5] T. Døssing, B. Herskind, S. Leoni, A. Bracco, R.A. Broglia, M. Matsuo, E. Vigezzi, Phys. Rep. 268 (1996) 1.

[6] T. Døssing, B. Herskind, A. Maj, M. Matsuo, E. Vigezzi, A. Bracco, S. Leoni, R.A. Broglia, Acta Phys. Pol. B32 (2001) 2565.

[7] P. Bosetti, S. Leoni, A. Bracco, B. Herskind, T. Døssing, G.B. Hagemann, R. Bark, A. Brocksted, P. Ekström, H. Carlsson, A. Nordlund, H. Ryde, F. Camera, S. Frattini, M. Mattiuzzi, B. Million, D. Bazzacco, R. Burch, G. de Angelis, D. De Acuna, M. de Poli, P. Pavan, Phys. Rev. Lett. 76 (1996) 1204.

[8] B.R. Mottelson, Nucl. Phys. A557(1993) 717c.

[9] O. Bohigas, H.A. Weidenmüller, Ann. Rev. Nucl. Part. Sci. 38 (1988) 421.

[10] H.A. Weidenmüller, Nucl. Phys. A520(1990) 509c.

[11] V. Zelevinsky, B.A. Brown, N. Frazier, M. Horoi, Phys. Rep. 276 (1996) 85.

[12] T.A. Brody, J. Flores, J.B. French, P.A. Mello, A. Pandey, S.S.M. Wong, Rev. Mod. 
Phys. 53(1981) 385.

M.L. Mehta, Random matrices, 2nd ed. (Academic Press, 1991).

[13] B.R. Barrett, R.F. Casten, J.N. Ginocchio, T. Seligman, H.A. Weidenmüller, Phys. Rev. C45 (1992) R1417.

[14] B. Mottelson, in The frontier of nuclear spectroscopy, eds. Y. Yoshizawa, H. Kusakari and T. Otsuka, (World Scientific 1993) p.7.

[15] M. Matsuo, T. Døssing, E. Vigezzi, and R. A. Broglia, Phys. Rev. Lett. 70 (1993) 2694.

[16] S. Åberg, Phys. Rev. Lett. 64 (1990) 3119.

S. Åberg, Prog. Nucl. and Part. Phys. 28 (1992) 11.

[17] M. Matsuo, T. Døssing, E. Vigezzi, S. Åberg, Nucl. Phys. A620 (1997) 296.

[18] J. Rekstad, T.S. Tveter, M. Guttormsen, Phys. Rev. Lett. 65(1990)2122; 67(1991)2236. R.K. Sheline, L. Bergholt, M. Guttormsen, J. Rekstad, T.S. Tveter, Phys. Rev. C51(1995)3078.

I. Huseby, T.S. Tveter, L. Bergholt, M. Guttormsen, E. Melby, J. Rekstad, S. Siem, R.K. Sheline, Phys. Rev. C55 (1997) 1805.

[19] P.G. Hansen, Phys. Rev. Lett. 67(1991)2235.

[20] V.G. Soloviev, Phys. Lett. B317(1993) 501.

[21] M. Matsuo T. Døssing, E. Vigezzi, R.A. Broglia, K. Yoshida, Nucl. Phys. A617 (1997) 1.

[22] R. Bengtsson, S. Frauendorf, Nucl. Phys. A314 (1979) 27; A327 (1979) 137.

[23] I.M. Green, S.A. Moszkowski, Phys. Rev. 139 (1965)B790.

[24] B. Lauritzen, T. Døssing, R.A. Broglia, Nucl. Phys. A457 (1986) 61.

[25] S. Frauendorf, Nucl. Phys. A557(1993) 259c. 
[26] A. Brockstedt, J. Lyttkens-Lindén, M. Bergström, L.P. Ekström, H. Ryde, J.C. Bacelar, J.D. Garrett, G.B. Hagemann, B. Herskind, F.R. May, P.O. Tjøm, S. Frauendorf, Nucl. Phys. A571(1994) 337.

[27] J.M. Espino, G.B. Hagemann, I.G. Bearden, R.A. Bark, M Bergström, A. Bracco, B. Herskind, H.J. Jensen, S. Leoni, C. Martínez-Torre, B. Million, P.O. Tjøm, Nucl. Phys. A640 (1998) 163.

[28] H.J. Jensen, G.B. Hagemann, P.O. Tjøm, S. Frauendorf, A. Atac,, M. Bergström, A. Bracco, A. Brockstedt, H. Carlsson, P. Ekström, J.M. Espino, B. Herskind, F. Ingebretsen, J. Jongman, S. Leoni, R.M. Lieder, T. Lönnroth, A. Maj, B. Million, A. Nordlund, J. Nyberg, M. Piiparinen, H. Ryde, M. Sugawara, A. Virtanen, Zeit. Phys. A340 (1991) 351 .

[29] A. Harsmann, Ph.D thesis, University of Copenhagen, 1998.

[30] A. Bracco, P. Bosetti, S. Frattini, E. Vigezzi, S. Leoni, T. Døssing, B. Herskind, M. Matsuo, Phys. Rev. Lett. 76 (1996) 4484. 


\section{FIGURES}

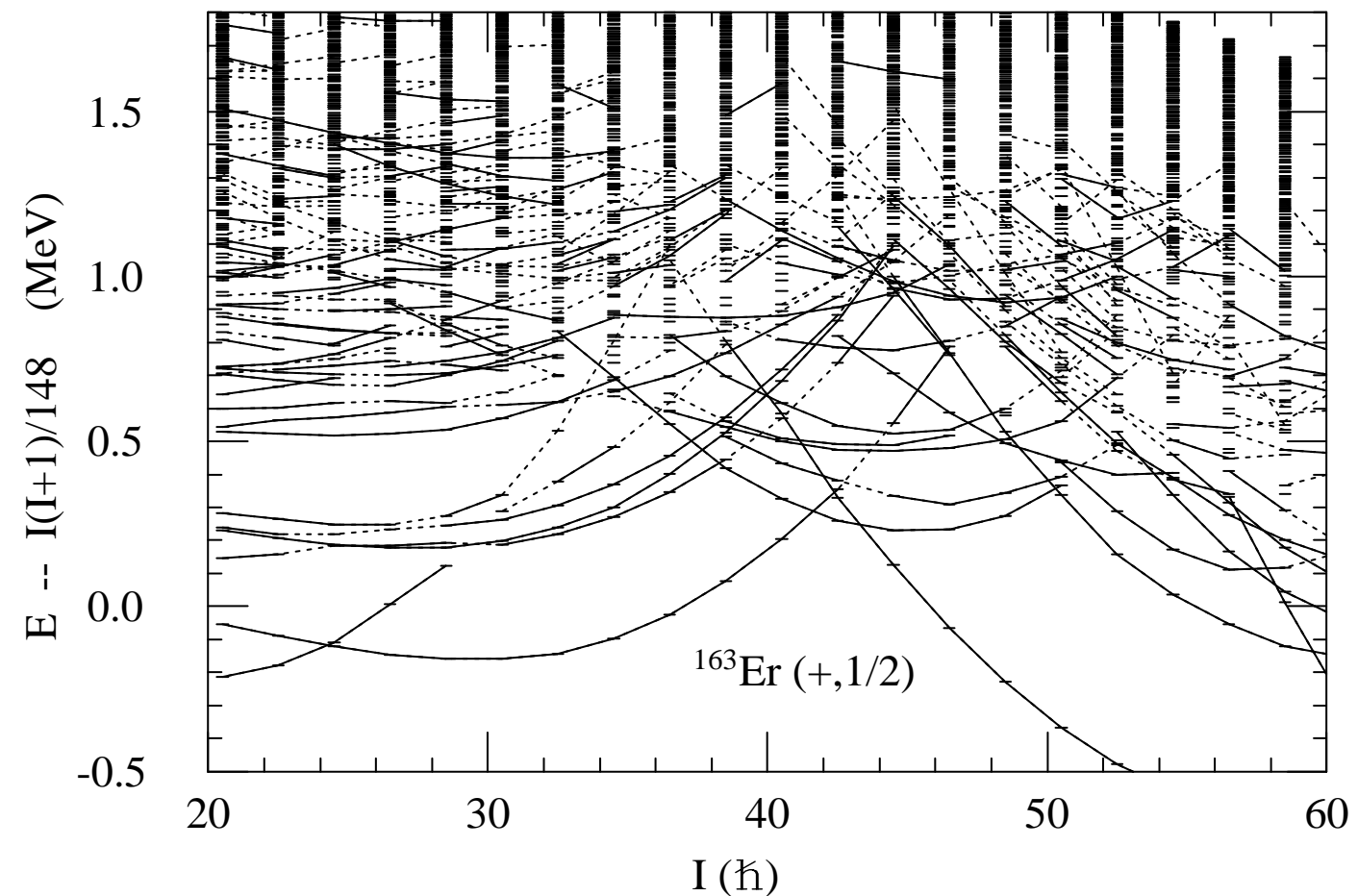

FIG. 1. The calculated energy levels in ${ }^{163}$ Er with parity and signature $(\pi, \alpha)=(+, 1 / 2)$. A reference rotational energy $I(I+1) / 148 \mathrm{MeV}$ is subtracted. The solid (dotted) lines connecting energy levels indicate stretched E2 transitions exhausting more than $71 \%$ (50\%) of the full rotational E2 strength. 


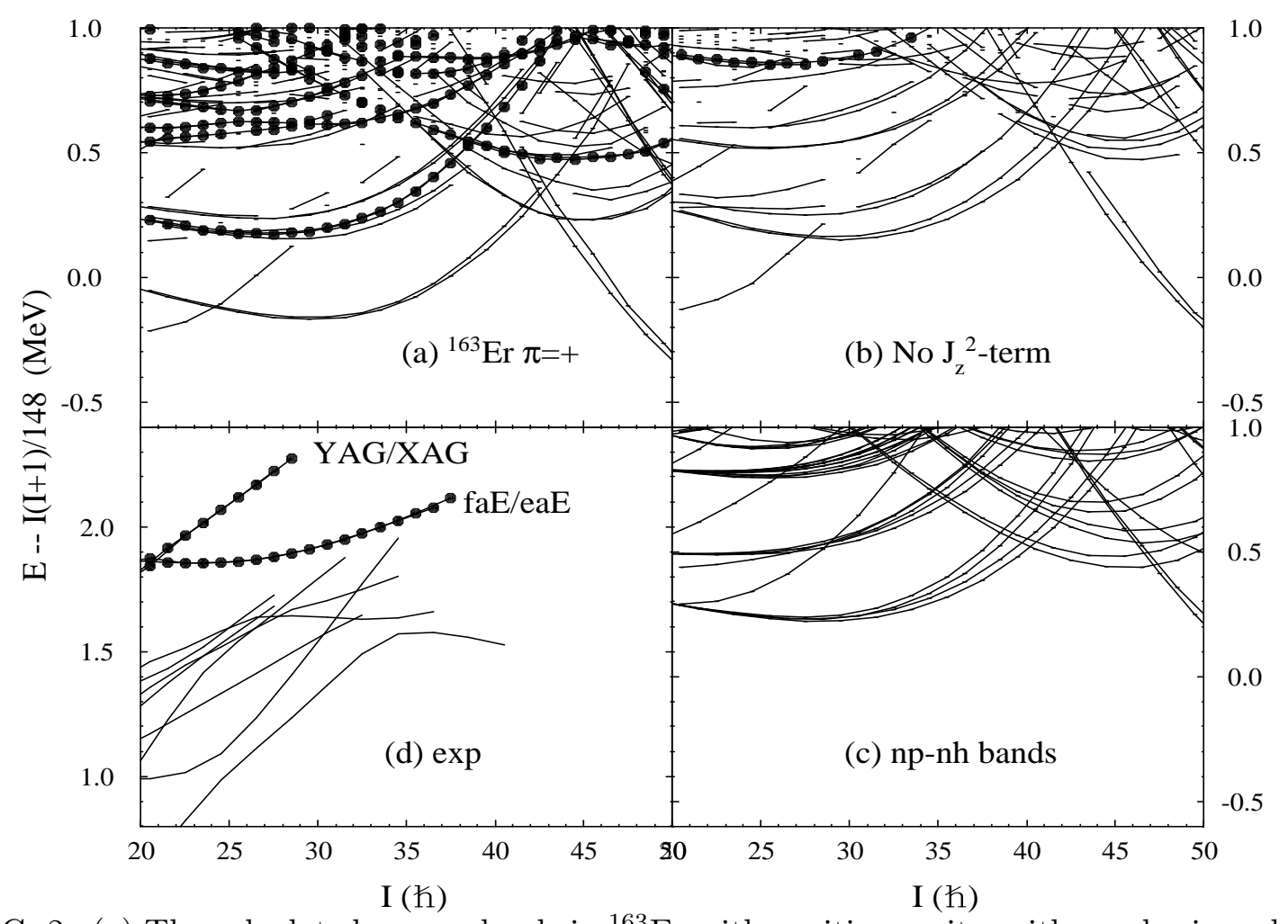

FIG. 2. (a) The calculated energy levels in ${ }^{163} \mathrm{Er}$ with positive parity, with emphasis on high-K states with $K_{i}>8$ marked with the filled circle. Both signatures $\alpha= \pm 1 / 2$ are included. The solid line connects the E2 transitions with more than $71 \%$. (b) The same as (a), but the $J_{z}^{2}$-term is neglected. (c) The same as (a), but the unperturbed np-nh cranked mean-field bands are displayed. (d) The experimental rotational bands with positive parity[1].The high-K bands are marked with the filled circle, and the labels represent the configurations assigned in the experiment (see text). 

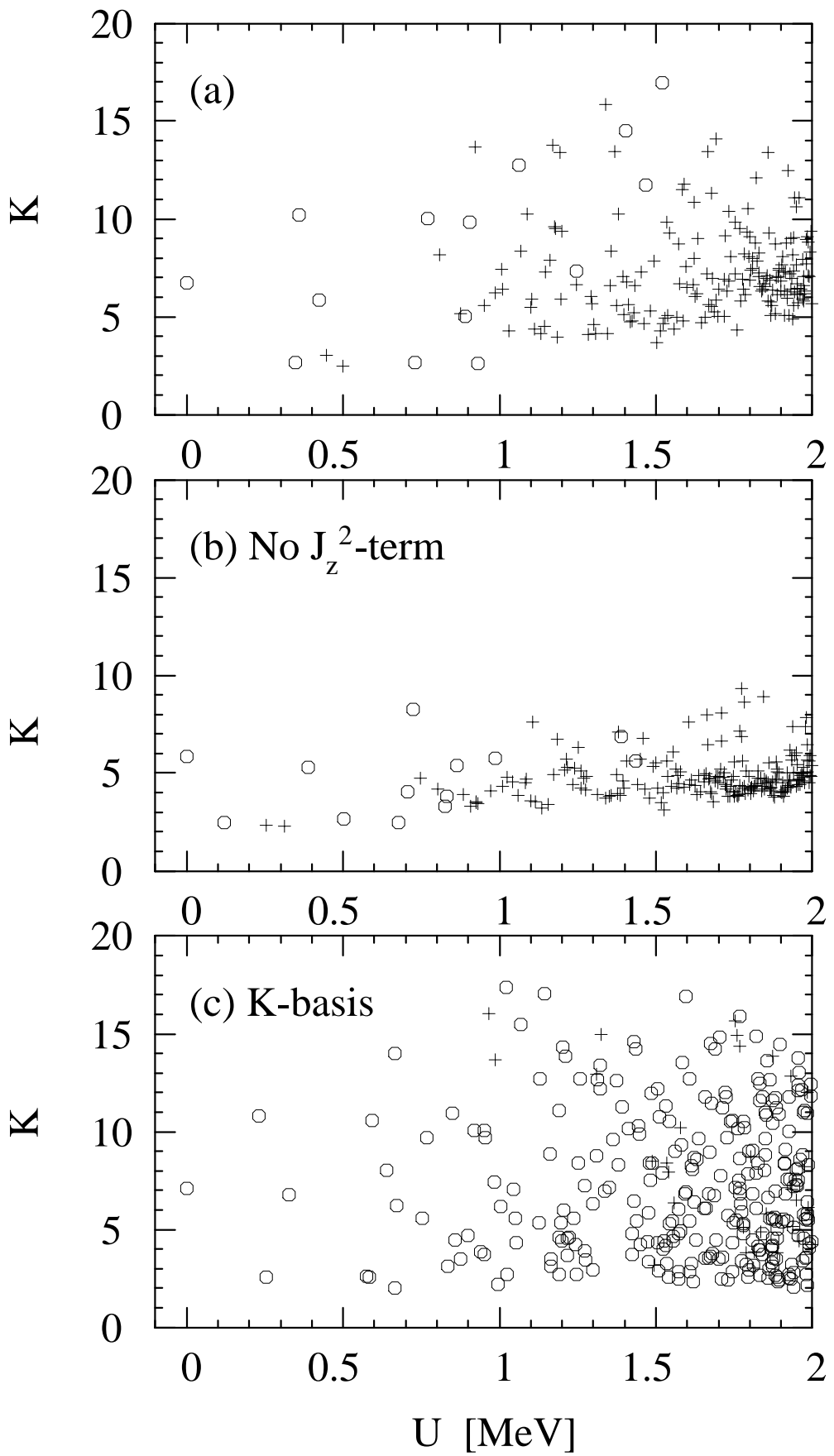

FIG. 3. The $\mathrm{K}$ values of the calculated states with ${I^{\pi}}^{\pi}=\frac{61}{2}^{+}$. The states forming rotational bands are marked with open circle, whereas the states with rotational damping are shown by crosses. (a) The full shell model Hamiltonian including the $J_{z}^{2}$-term is diagonalized. (b) The $J_{z}^{2}$-term is neglected. (c) The $J_{z}^{2}$-term is kept but the residual two-body interaction is neglected to produce the K-basis states. 

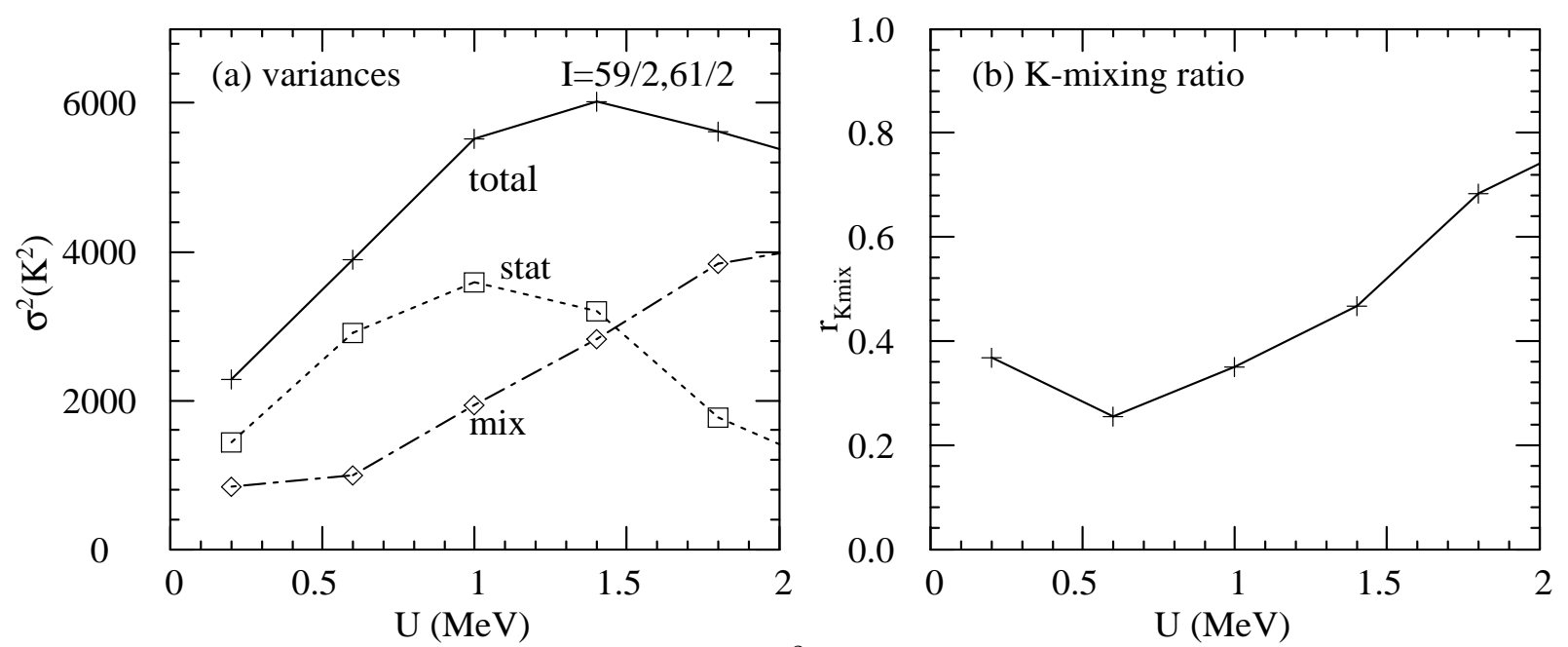

FIG. 4. (a)The variances with respect to $J_{z}^{2}$ calculated for energy bins $0.4 \mathrm{MeV}$ wide, for spins $I=59 / 2,61 / 2$ including both parities. The solid, dotted and dashed lines indicate $\sigma^{2}\left(K^{2}\right)_{t o t}, \sigma^{2}\left(K^{2}\right)_{\text {stat }}$, and $\sigma^{2}\left(K^{2}\right)_{m i x}$, respectively. In this calculation, 10000 basis states are used for diagonalization. (b) The K-mixing ratio $r_{K m i x}$ calculated for the same energy bins. 

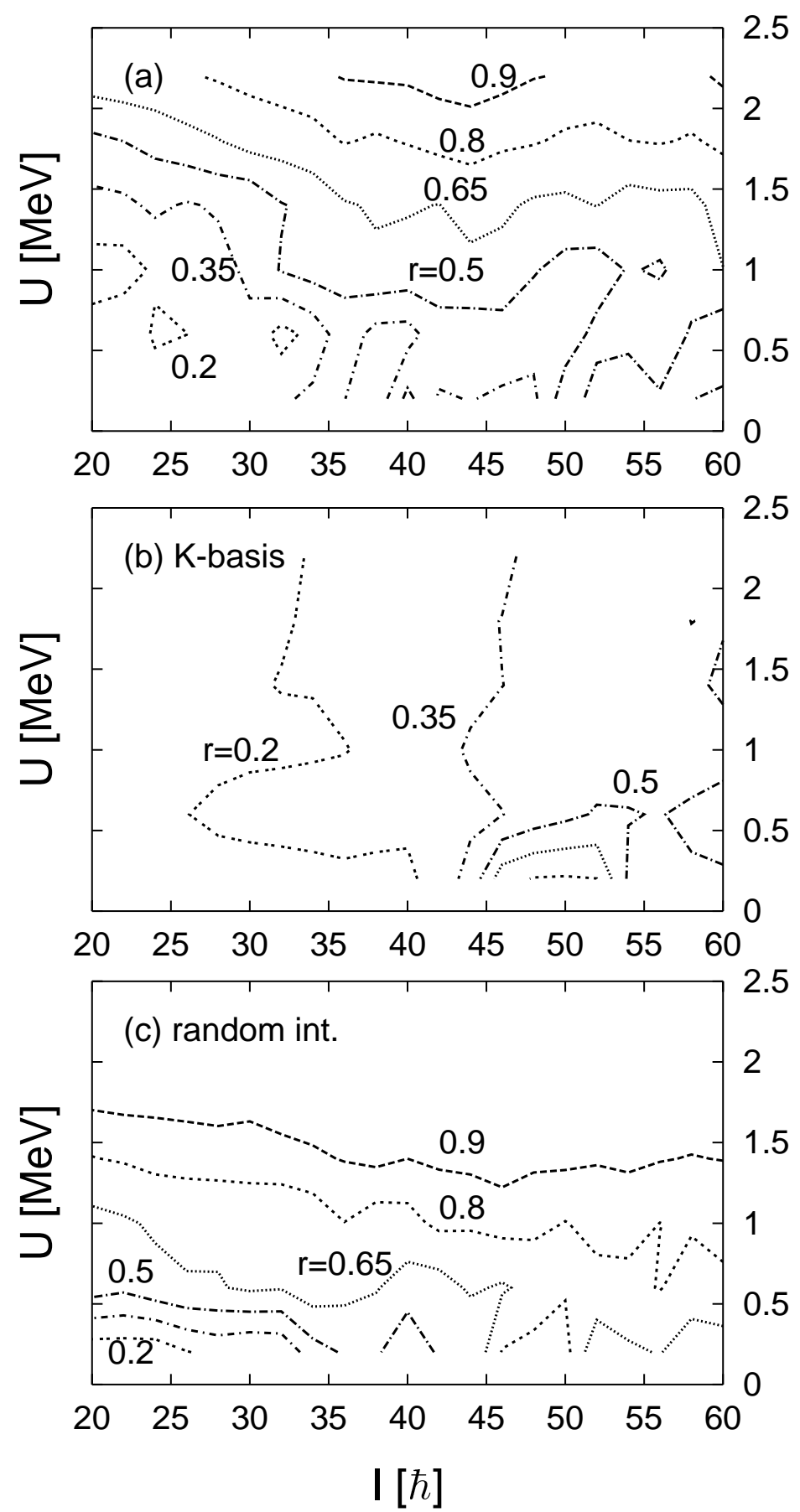

FIG. 5. (a) Contour plot of the K-mixing ratio $r_{K m i x}$ as a function of spin $I$ and thermal excitation energy $U$. (b) The same as (a), but the residual SDI is neglected. (c) The same as (a), but the residual SDI is replaced with a random two-body interaction. 

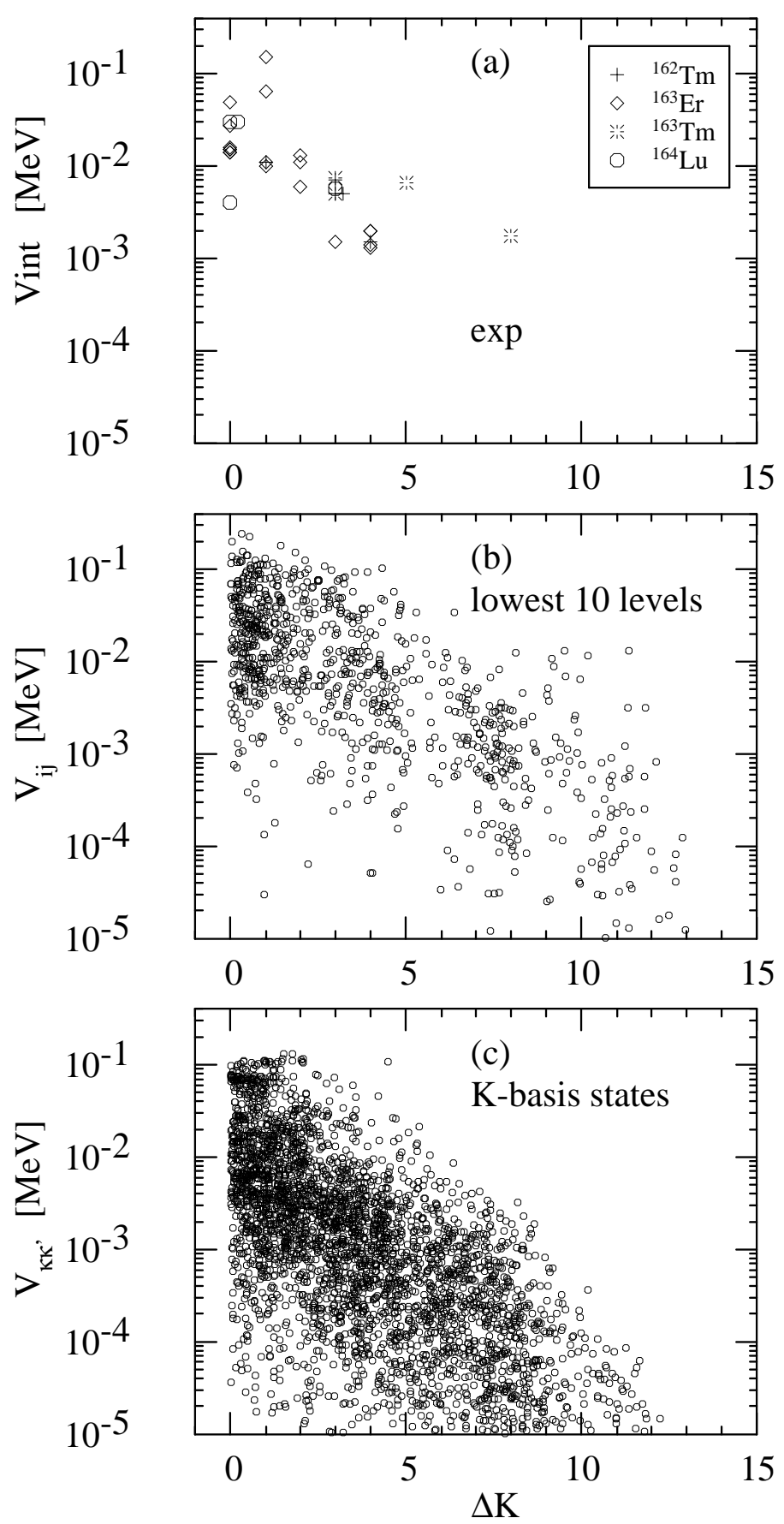
FIG. 6. (a) The experimentally extracted interaction strengths at band crossings, correlated with the difference $\Delta K$ between the band-head K-quantum numbers of the crossing bands (see text). (b) The calculated matrix elements $V_{i j}$ of the residual SDI among the lowest energy levels(10 for each $I^{\pi}$ ), which approximately correspond to the states forming discrete rotational bands, where $V$ is its absolute size while $\Delta K$ is the difference of $\mathrm{K}$ values of two energy levels. Those calculated for $I^{\pi}=39 / 2^{ \pm}-57 / 2^{ \pm}$are collected. (c) The calculated matrix elements $V_{\kappa \kappa^{\prime}}$ among the lowest 20 K-basis states. 


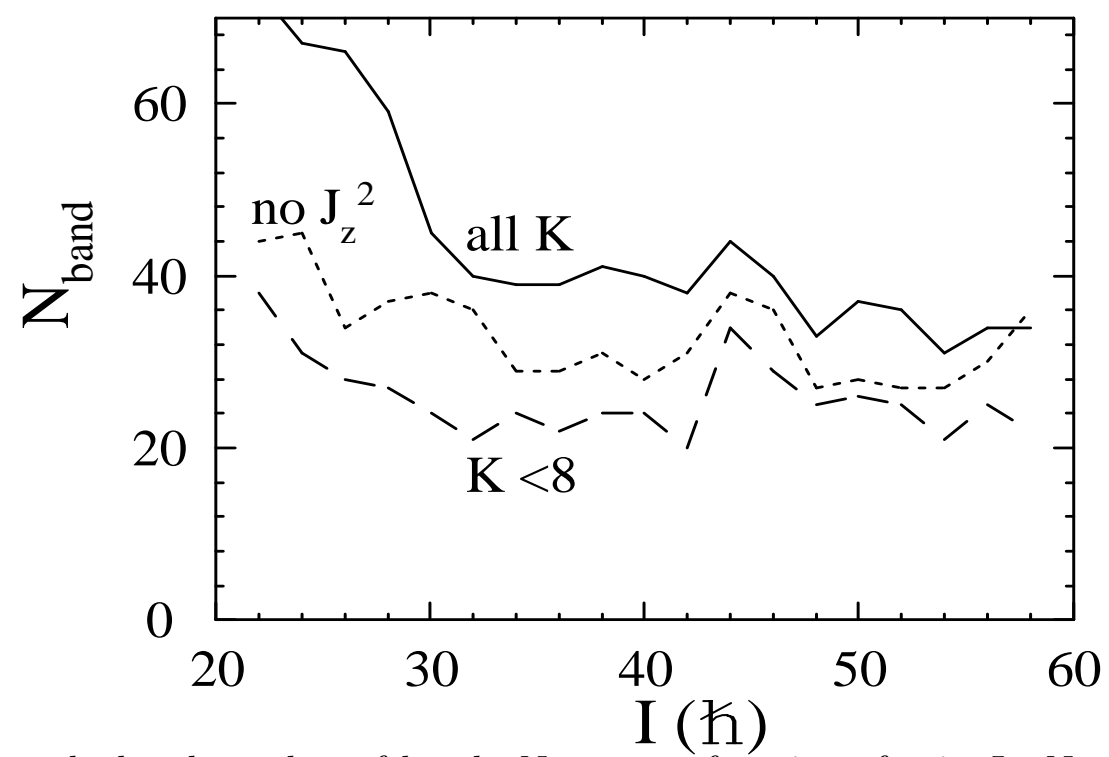

FIG. 7. The calculated number of bands $N_{\text {band }}$ as a function of spin $I . N_{\text {band }}$ for the states with $K<8$ is plotted by the dashed curve. $N_{\text {band }}$ calculated without the $J_{z}^{2}$-term is also shown with dotted curve. 